\title{
Members of the Floating Pool Crew
}

\section{NYC CREW}

Adrian Benepe, Commissioner, New York City Department of Parks, 2002-12

Michael Bloomberg, Mayor of New York City, 2002-14

Bill de Blasio, Mayor of New York City, 2014-present*

Daniel Doctoroff, Deputy Mayor for Economic Development and Rebuilding, 2002-8

Liam Kavanagh, First Deputy Commissioner, New York City Department of Parks, 2002-present ${ }^{*}$

Joshua Laird, Director of Planning; Assistant Commissioner for Planning and Parklands, New York City Department of Parks, 1997-2013

Robert Moses, Commissioner, New York City Department of Parks, 1934-60

* Office held as of the writing of this book. 
Mitchell Silver, Commissioner, New York City Department of Parks, 2014-present ${ }^{*}$

Henry J. Stern, Commissioner, New York City Department of Parks, 1983-90, 1994-2000

David Yassky, Member, New York City Council, 2002-9

\section{NYS CREW}

Richard Dorado, Senior Counsel, Empire State Development Corporation

Udo Drescher, Assistant Regional Attorney for New York State Department of Environmental Conservation, Region 2

Pat Foye, Downstate Chairman, Empire State Development Corporation, 2007-8

Pete Grannis, Commissioner, New York State Department of Environmental Conservation, 2007-10

Stuart Gruskin, Executive Deputy Commissioner, New York State Department of Environmental Conservation, 2007-10; Staff of New York State Department of Environmental Conservation, Region 2, 2000-2007

Rose Keville, Consultant to the Empire State Development Corporation and the Brooklyn Bridge Park Development Corporation, 2007

Marianna Koval, Executive Director, Brooklyn Bridge Park Coalition (renamed Brooklyn Bridge Park Conservancy), 1998-2009

Wendy Leventer, President, Brooklyn Bridge Park Development Corporation, 2004-7

Suzanne Mattei, Director, New York State Department of Environmental Conservation, Region 2, 2007-10

* Office held as of the writing of this book. 
Regina Myer, President, Brooklyn Bridge Park Development Corporation (renamed Brooklyn Bridge Park Corporation), 2007-16

Jennifer Rimmer, Vice President for Subsidiary Development, Brooklyn Bridge Park Development Corporation, 2007-9

Avi Schick, President and Chief Operating Officer, Empire State Development Corporation, 2007-9

Eliot Spitzer, Governor of New York State, 2007-8

\section{HOBOKEN CREW}

Jack Carbone, Attorney

Robert Drasheff, Director, Waterfront Development

L. Michael Krieger, Port Authority of New York and New Jersey:

Manager, Hoboken Waterfront Development, and General

Manager, Regional and Economic Development; Hoboken: Special

Waterfront Counsel

David Roberts, Mayor of Hoboken, 2001-9

Anthony Russo, Mayor of Hoboken, 1993-2001

Cassandra Wilday, Landscape Architect

\section{TEAM NEPTUNE}

Kent Barwick, Neptune Board Member

Lawrence B. Buttenwieser, Neptune Board Member

Svein Christofferson, Maritime Attorney, Holland \& Knight

Carter Craft, Metropolitan Waterfront Alliance

Steven Crainer, Attorney, Rosenman \& Colin; Seyfarth Shaw

Charles Cushing, Naval Engineer, C. R. Cushing \& Company 
John Keenan, Insurance Agent, Keenan Marine Insurance Agency

Jonathan Kirschenfeld, Architect, Jonathan Kirschenfeld Architect, PC

Malcolm McLaren, Maritime Engineer, McLaren Engineering Group

Kent Merrill, Naval Architect and Marine Engineer, Project

Manager, C. R. Cushing \& Company

Johann Mordhorst, Associate, Jonathan Kirschenfeld Consultants

Steven Sivak, Construction Manager

Brian Starer, Attorney, Holland \& Knight

Tillett Lighting, Lighting Consultants

Joe Tortorella, Engineer, Robert Sillman Associates, Structural Engineers

Joel Trace, Architect, Swimming Pool Consultant

Truax \& Company, Translucent Murals

\section{OTHERS}

Eric Bernholz, Chief, United States Coast Guard Inspections Division Edith K. Ehrman, Benefactor

Stephen Kass, Senior Environmental Counsel, Carter Ledyard \& Milburn

Steve Kass, President, American Leisure Corp.

Lillian Liburdi (Borrone), Director of Port Commerce, Port Authority of New York and New Jersey Joe Mizzi, President, Sciame Construction Company

Frank Sciame, Chairman/CEO, Sciame Construction Company

Paul Seck, Landscape Architect, Michael Van Valkenburgh Associates 


\section{The Floating Pool Lady}

